\title{
CORNER VIEW ON THE CROWN DOMAIN
}

\author{
BERNHARD KRÖTZ
}

Date: June 19, 2021. 


\section{Introduction}

This paper is about the crown domain, henceforth denoted by $\Xi$, which is the canonical complexification of a Riemmanian symmetric space $X$ of the non-compact type. Specifically we are interested in the the nature of the boundary of $\Xi$ and eventually in good compactifications of $\Xi$.

Let us begin with some possible definitions of $\Xi$. Let us denote by $G$ the connected component of the isometry group of $X$. Then

$$
X=G / K
$$

for $K<G$ a maximal compact subgroup. Group-theoretically one can view $X$ as the moduli space of all maximal compact subgroups of the semisimple group $G$.

Before we advance let us recall some examples.

- For $G=\operatorname{PSl}(2, \mathbb{R})$ it is custom to identify $X$ with the upper half plane $\mathbf{H}=\{z \in \mathbb{C} \mid \operatorname{Im} z>0\}$. Let us refer to [6] for a comprehensive discussion of the corresponding crown domain and the analysis thereon.

- For $G=\operatorname{PSl}(n, \mathbb{R})$ one can view $X$ as the space of positive definite unimodular matrices, i.e. $X=\operatorname{Sym}(n, \mathbb{R})_{\operatorname{det}=1}^{+}$.

- For $G=\operatorname{PSp}(n, \mathbb{R})$ one often realizes $X$ as the Siegel domain $V+i \Omega$ with $V=\operatorname{Sym}(n, \mathbb{R})$ and $\Omega \subset V$ the cone of positive definite matrices.

Next we discuss complexifications of $X$. By a complexification we simply mean a connected complex manifold $\Xi$ which contains $X$ as a totally real submanifold. We wish to request that complexification respects symmetry, i.e. the action of $G$ on $X$ extends to $\Xi$.

The natural candidate for a complexification seems to be $X_{\mathbb{C}}=$ $G_{\mathbb{C}} / K_{\mathbb{C}}$ with $G_{\mathbb{C}}$ and $K_{\mathbb{C}}$ the universal complexifications of $G$ and $K$ respectively. We often call $X_{\mathbb{C}}$ the affine complexification of $X$ as it is an affine variety. For example for $X=\operatorname{Sym}(n, \mathbb{R})_{\operatorname{det}=1}^{+}$one has $X_{\mathbb{C}}=\operatorname{Sym}(n, \mathbb{C})_{\operatorname{det}=1}$.

Let us be more demanding on our complexification and request that the Riemannian metric of $X$ extends to a $G$-invariant metric on $\Xi$ (see [9], Sect. 4). This is now a severe restriction on $\Xi$ as it forces the $G$-action on $\Xi$ to be proper. For instance $G$ does not act properly on $X_{\mathbb{C}} 1$ and this guides us to smaller $G$-domains in $X_{\mathbb{C}}$ where $G$ acts properly. The study of proper $G$-actions on $X_{\mathbb{C}}$ began in [1] and a complete classification of all maximal open $G$-neighborhoods of $X$ in

\footnotetext{
${ }^{1}$ The $G$-stabilizer of $\operatorname{diag}(i,-i) \in \operatorname{Sym}(2, \mathbb{C})_{\operatorname{det}=1}$ is $\operatorname{PSO}(1,1)$ and not compact.
} 
$X_{\mathbb{C}}$ with proper action was obtained in [7]. In view of the results of [7] one can define the crown domain as the intersection of all maximal open $G$-neighborhoods of $X$ in $X_{\mathbb{C}}$ with proper action ([7).

Let us give another construction of $\Xi$. Denote by $T X$ the tangent bundle of $X$. This is a homogeneous vector bundle over $X$ on which $G$ acts properly. There is a natural $G$-map from $T X$ to $X_{\mathbb{C}}$ (see [9], Sect. 4 ) and the crown domain corresponds to the maximal $G$-neighborhood of $X$ in $T X$ which embeds into $X_{\mathbb{C}}$.

A third and perhaps prefered way to define the crown domain is as the universal domain for holomorphically extended orbit maps of unitary spherical representations of $G$ (see the introduction of [8]).

Let us turn now to the subject proper of this paper, the topological boundary $\partial \Xi$ of $\Xi$ in $X_{\mathbb{C}}$. The boundary is a very complicated object and there is little hope to obtain an explicite description. However $\partial \Xi$ features some structure; for instance, in it one finds the distinguished boundary $\partial_{d} \Xi \subset \partial \Xi$, introduced in [3]. The distinguished boundary is some sort of Shilov boundary of $\Xi$ in the sense that it is the smallest closed subset in $\partial \Xi$ on which bounded plurisubharmonic functions on $\operatorname{cl}(\Xi)$ attend their maximum.

We know from [3] and [8] that $\partial_{d} \Xi$ is a finite (and explicite) union of $G$-orbits, say

$$
\partial_{d} \Xi=\mathcal{O}_{1} \amalg \ldots \amalg \mathcal{O}_{s} .
$$

From now on we shall identify each $\mathcal{O}_{j}$ with a homogeneous space: $G / H_{j}$. The main result of [3] was:

If $G / H_{j}$ is a symmetric space, then it is a non-compactly causal symmetric space. Moreover, every non-compactly causal symmetric space $Y=G / H$ appears in the distinguished boundary of the corresponding crown domain for $X=G / K$.

One aim of this paper is to understand this result better. To be more concise: what is the reason that precisely non-compactly causal (NCC) symmetric spaces appear in the boundary? As we will see, answering this question will eventually reveal the structure of $\partial \Xi$.

NCC-spaces are very special among all semisimple symmetric spaces. We recall their definition (see [5]). We assume the Lie algebra of $G$ to be simple and write $\mathfrak{q}$ for the tangent space of $Y$ at the standard base point $y_{o}=H \in Y$. We note that $\mathfrak{q}$ is a linear $H$-module. Now, noncompactly causal means that $\mathfrak{q}$ admits an non-empty open $H$-invariant convex cone, say $C$, which is hyperbolic and does not contain any affine lines. 
The theme of this paper is to view $\Xi$ from the corner point $y_{o} \in Y$ and not as a thickening of $X$ as in the customary definitions from above. Now a slight precisioning of terms is necessary. As we saw, $\partial_{d} \Xi$ might have several connected components. If this happens to be the case, then we shrink $\Xi$ to a $G$-domain $\Xi_{H}$ whose distinguished boundary is precisely $Y$, see [4].

For $C \subset \mathfrak{q}$ the minimal cone (see [5]) we form in the tangent bundle $T Y=G \times_{H} \mathfrak{q}$ the cone-subbundle

$$
\mathcal{C}=G \times{ }_{H} C
$$

and with that its boundary cone-bundle

$$
\partial \mathcal{C}=G \times_{H} \partial C .
$$

In this context we ask the following

Question: Is there a G-equivariant, generically injective, proper continuous surjection $p: \partial \mathcal{C} \rightarrow \partial \Xi_{H}$ ?

In other words, we ask if there exists an equivariant "resolution" of the boundary in terms of the geometrically simple boundary cone bundle $\partial \mathcal{C}$.

In this paper we give an affirmative answer to this question if $X$ is a Hermitian tube domain. In this simplified situation the crown domain is $\Xi=X \times \bar{X}$ with $\bar{X}$ denoting $X$ but endowed with the opposite complex structure (i.e., if $X$ is already complex, then the crown is the complex double). On top of that $\partial_{d} \Xi=Y$ is connected, i.e. $\Xi=\Xi_{H}$.

I wish to point out that the presented method of proof will not generalize. In order to advance one has to understand more about the structure of the minimal cone $C$; one might speculate that some sort of " $H \cap K$-invariant theory" for $C$ could be useful.

Let me pose two open problems:

Problem 1: For general $\Xi$, does $\partial \Xi_{H}$ admit a resolution as a cone bundle in the sense described above.

Problem 2: Construct $G$-equivariant compactification of $\Xi$, resp. $\Xi_{H}$.

Acknowledgement: The origin of this paper traces back to my productive stay at the RIMS in 2005/2006. I am happy to express my gratitude to my former host Toshiyuki Kobayashi. Also I would like to thank Toshihiko Matsuki for some useful intuitive conversations arround this topic. 


\section{Main part}

Let $X=G / K$ be a Hermitian symmetric space of tube type. This means that there is an Euclidean (or formally real) Jordan algebra $V$ with positive cone $W \subset V$ such that

$$
X=V+i W \subset V_{\mathbb{C}} .
$$

The action of $G$ is by fractional linear transformation and our choice of $K$ is such it fixes the base point $x_{0}=i e$ with $e \in V$ the identity element of the Jordan algebra.

It is no loss of generality if we henceforth restrict ourselves to the basic case of $G=\operatorname{Sp}(n, \mathbb{R})$ - the more general case is obtained by using standard dictionary which can be found in text books, e.g. 22.

For our specific choice, the Jordan algebra is $V=\operatorname{Sym}(n, \mathbb{R})$ and $W \subset V$ is the cone of positive definite symmetric matrices. The identity element $e$ is $I_{n}$, the $n \times n$ identity matrix. The group $G$ acts on $X$ by standard fractional linear transformations: $g=\left(\begin{array}{ll}a & b \\ c & d\end{array}\right) \in G$ with appropriate $a, \ldots, d \in M(n, \mathbb{R})$ acts as

$$
g \cdot z=(a z+b)(c z+d)^{-1} \quad(z \in X) .
$$

The maximal compact subgroup $K$ identifies with $U(n)$ under the standard embedding

$$
U(n) \rightarrow G, \quad u+i v \mapsto\left(\begin{array}{cc}
u & v \\
-v & u
\end{array}\right) \quad(u, v \in M(n, \mathbb{R}))
$$

It is then clear that $K=U(n)$ is the stabilizer of $x_{0}=i I_{n}$. In the sequel we consider $V_{\mathbb{C}}$ as an affine piece of the projective variety $\mathcal{L}$ of Lagrangians in $\mathbb{C}^{2 n}$; the embedding is given by

$$
V_{\mathbb{C}} \mapsto \mathcal{L}, \quad T \mapsto L_{T}:=\left\{(T(v), v) \mid v \in \mathbb{C}^{n}\right\} .
$$

It is then clear that $G_{\mathbb{C}}=\operatorname{Sp}(n, \mathbb{C})$ acts on $\mathcal{L}$; in symbols: $g=$ $\left(\begin{array}{ll}a & b \\ c & d\end{array}\right) \in G_{\mathbb{C}}$ with appropriate $a, \ldots, d \in M(n, \mathbb{C})$ acts as

$$
g \cdot L=\{(a v+b w, c v+d w) \mid(v, w) \in L\} \quad(L \in \mathcal{L}) .
$$

The space $\mathcal{L}$ is homogeneous under $G_{\mathbb{C}}$. If we choose the base point

$$
x_{0} \leftrightarrow L_{0}=\left\{(i v, v) \mid v \in \mathbb{C}^{n}\right\},
$$

then the stabilizer of $x_{0}$ in $G_{\mathbb{C}}$ is the Siegel parabolic

$$
S^{+}=K_{\mathbb{C}} \ltimes P^{+} \quad \text { and } \quad P^{+}=\left\{\mathbf{1}+\left(\begin{array}{cc}
u & -i u \\
-i u & -u
\end{array}\right) \mid u \in V_{\mathbb{C}}\right\} .
$$


Thus we have

$$
\mathcal{L}=G_{\mathbb{C}} \cdot L_{0} \simeq G_{\mathbb{C}} / S^{+}
$$

Sometimes it is useful to take the conjugate base point $\overline{x_{0}}=-i I_{n}$. Then the stabilizer of $\overline{L_{0}}$ in $\mathcal{L}$ is the oppposite Siegel parabolic

$$
S^{-}=K_{\mathbb{C}} \ltimes P^{-} \quad \text { and } \quad P^{-}=\left\{\mathbf{1}+\left(\begin{array}{cc}
u & i u \\
i u & -u
\end{array}\right) \mid u \in V_{\mathbb{C}}\right\}
$$

and

$$
\mathcal{L}=G_{\mathbb{C}} \cdot \overline{L_{0}} \simeq G_{\mathbb{C}} / S^{-}
$$

Next we come to the realization of the affine complexification $X_{\mathbb{C}}=$ $G_{\mathbb{C}} / K_{\mathbb{C}}$. We consider the $G_{\mathbb{C}^{-}}$equivariant embedding

$$
X_{\mathbb{C}} \rightarrow \mathcal{L} \times \mathcal{L}, \quad g K_{\mathbb{C}} \mapsto\left(g \cdot L_{0}, g \cdot \overline{L_{0}}\right) .
$$

It is not hard to see that

$$
X_{\mathbb{C}}=\left\{\left(L, L^{\prime}\right) \in \mathcal{L} \times \mathcal{L} \mid L+L^{\prime}=\mathbb{C}^{2 n}\right\},
$$

i.e., $X_{\mathbb{C}}$ is the affine variety of pairs of transversal Lagrangians.

Set $\bar{X}=V-i W$ and note that the map $z \mapsto \bar{z}$ identifies $X$ with $\bar{X}$ in a $G$-equivariant, but antiholomorphic manner.

Next we come to the subject matter, the crown domain of $X$ :

$$
\Xi=X \times \bar{X} \subset X_{\mathbb{C}}
$$

Let us denote by $\partial \Xi$ the topological boundary of $\Xi$ in $X_{\mathbb{C}}$. The goal is to resolve $\partial \Xi$ by a cone bundle over the affine symmetric space $Y=G / H$ where $H=\operatorname{Gl}(n, \mathbb{R})$ is the structure group of the Euclidean Jordan algebra $V$.

We define an involution $\tau$ on $G$ by

$$
\tau(g)=I_{n, n} g I_{n, n} \quad \text { where } \quad I_{n, n}=\left(\begin{array}{ll}
I_{n} & \\
& -I_{n}
\end{array}\right) .
$$

The fixed point set of $\tau$ is

$$
H=\left\{\left(\begin{array}{ll}
a & \\
& a^{-t}
\end{array}\right) \mid a \in \operatorname{Gl}(n, \mathbb{R})\right\}=\operatorname{Gl}(n, \mathbb{R}) .
$$

We write $\mathfrak{h}$ for the Lie algebra of $H$ and denote by $\tau$ as well the derived involution on $\mathfrak{g}$. The $\tau$-eigenspace decomposition on $\mathfrak{g}$ shall be denoted by

$$
\mathfrak{g}=\mathfrak{h}+\mathfrak{q} \quad \text { where } \quad \mathfrak{q}=\left(\begin{array}{cc}
0 & V \\
V & 0
\end{array}\right)
$$


Write $\mathfrak{q}^{+}=\left(\begin{array}{cc}0 & V \\ 0 & 0\end{array}\right)$ and $\mathfrak{q}^{-}=\left(\begin{array}{cc}0 & 0 \\ V & 0\end{array}\right)$ and note that

$$
\mathfrak{q}=\mathfrak{q}^{+} \oplus \mathfrak{q}^{-}
$$

is the splitting of $\mathfrak{q}$ into two inequivalent irreducible $H$-modules.

The affine space $Y=G / H$ admits (up to sign) a unique $H$-invariant convex open cone $C \subset \mathfrak{q}$, containing no affine lines and consisting of hyperbolic elements. Explicitely:

$$
C=\left(\begin{array}{cc}
0 & W \\
W & 0
\end{array}\right)=W \oplus W \subset \mathbf{q}^{+} \oplus \mathbf{q}^{-} .
$$

We form the cone bundle

$$
\mathcal{C}=G \times{ }_{H} C
$$

and note that there is a natural $G$-equivariant map

$$
P: G \times_{H} C \rightarrow \Xi, \quad\left[g,\left(y_{1}, y_{2}\right)\right] \mapsto g \cdot\left(i y_{1},\left(i y_{2}\right)^{-1}\right) .
$$

Let us verify that this map is in fact defined. For that one needs to check that for $h \in H$ and $y_{1}, y_{2} \in W$, the elements $\left(h, y_{1}, y_{2}\right)$ and $\left(\mathbf{1}, h y_{1} h^{t}, h^{-t} y_{2} h^{-1}\right)$ have the same image. Indeed,

$$
h \cdot\left(i y_{1},\left(i y_{2}\right)^{-1}\right)=\left(i h y_{1} h^{t}, h\left(i y_{2}\right)^{-1} h^{t}\right)=\left(i h y_{1} h^{t},\left(i h^{-t} y_{2} h^{-1}\right)^{-1}\right)
$$

which was asserted.

Lemma 2.1. The map $P: \mathcal{C} \rightarrow \Xi$ is onto.

Proof. Write $A$ for the group of diagonal matrices in $G$ with positive entries. Note that the Lie algebra $\mathfrak{a}$ of $A$ is a maximal flat in $\mathfrak{p}=$ $\mathfrak{g} \cap \operatorname{Sym}(2 n, \mathbb{R})$. In general, we know that $\mathfrak{p}=\operatorname{Ad}(K) \mathfrak{a}$. Furthermore, if $W_{d}$ denotes the diagonal part of $W$, then $i W_{d}=A \cdot x_{0}$. From $G=K A K$ it now follows that for any two points $(z, w) \in X$ there exist a $g \in G$ such that $g \cdot(z, w)=\left(x_{0}, w^{\prime}\right)$ with $w^{\prime} \in i W_{d}$. As a consequence we obtain that

$$
\Xi=G \cdot\left(i W_{d},-i I_{n}\right) .
$$

Clearly the right hand side is contained in the image of $P$ and this finishes the proof.

Remark 2.2. (a) The map $P$ is not injective. We shall give two different arguments for this assertion, beginning with an abstract one. If $P$ were injective, then $P$ establishes an homeoporphism between $\Xi$ and $\mathcal{C}=G \times{ }_{H} C$. In particular $\Xi$ is homotopy equivalent to $Y=G / H$. But we know that $\Xi$ is contractible; a contradiction. 
More concretely for $k \in K, k \neq \mathbf{1}$, the elements $\left[k,\left(i I_{n},-i I_{n}\right)\right] \neq$ $\left[\mathbf{1},\left(i I_{n},-i I_{n}\right)\right]$ have the same image in $\Xi$. It should be remarked however, that the map is generically injective.

(b) As $H$ acts properly on $C$, it follows that $G$ acts properly on the cone-bundle $G \times{ }_{H} C$. Further it is not hard to see that the map $P$ is proper.

We need a more invariant formulation of the map $P$. For that, note that the rational map

$$
V_{\mathbb{C}} \rightarrow V_{\mathbb{C}}, \quad z \mapsto-z^{-1}
$$

belongs to $K$. Its extension to $\mathcal{L}$, shall be denoted by $s_{0}$ and is given by

$$
s_{0}(L)=\left\{(-w, v) \in \mathbb{C}^{2 n} \mid(v, w) \in L\right\} .
$$

Also, the anti-symplectic map $V_{\mathbb{C}} \rightarrow V_{\mathbb{C}}, z \mapsto-z$ has a natural extension to $\mathcal{L}$ given by

$$
L \mapsto-L:=\left\{(-v, w) \in \mathbb{C}^{2 n} \mid(v, w) \in L\right\} .
$$

In this way, we can rewrite $P$ as

$$
P: G \times{ }_{H} C \rightarrow \Xi, \quad\left[g,\left(y_{1}, y_{2}\right)\right] \mapsto g \cdot\left(i y_{1},-s_{0}\left(i y_{2}\right)\right)
$$

and we see that $P$ extends to a continuous map

$$
\tilde{P}: G \times_{H} \mathfrak{q} \rightarrow \mathcal{L} \times \mathcal{L}, \quad\left[g,\left(y_{1}, y_{2}\right)\right] \mapsto g \cdot\left(i y_{1},-s_{0}\left(i y_{2}\right)\right) .
$$

We restrict $\tilde{P}$ to $G \times{ }_{H} \partial C$ and call this restriction $p$. It is clear that $\operatorname{im} p$ is contained in the boundary of $\Xi$ in $\mathcal{L} \times \mathcal{L}$. But even more is true: the following proposition constitutes a $G$-equivariant "resolution" of $\partial \Xi$.

Proposition 2.3. im $p \subset \partial \Xi$ and the G-equivariant map

$$
p: G \times_{H} \partial C \rightarrow \partial \Xi, \quad\left[g,\left(y_{1}, y_{2}\right)\right] \mapsto g \cdot\left(i y_{1},-s_{0}\left(i y_{2}\right)\right)
$$

is onto and proper.

Proof. We first show that $\operatorname{im} p \subset \partial \Xi$. This means that $\operatorname{im} p \subset X_{\mathbb{C}}$. In fact, from Lemma 2.1 and the definition of $p$ it follows that im $p$ is contained in the closure of $\Xi$ in $\mathcal{L} \times \mathcal{L}$ and does not intersect $\Xi$.

Let us now show that $\operatorname{im} p \subset X_{\mathbb{C}}$. First note that

$$
\partial C=W \times \partial W \amalg \partial W \times \partial W \amalg \partial W \times W .
$$

Thus the assertion will certainly follow if we verify the following slightly stronger statement: for $y_{1}, y_{2} \in c l(W)$ the Lagrangians

$$
L_{1}=\left\{\left(i y_{1} v, v\right) \mid v \in \mathbb{C}^{n}\right\} \quad \text { and } \quad L_{2}=\left\{\left(w, i y_{2} w\right) \mid w \in \mathbb{C}^{n}\right\}
$$


are transversal. We use the structure group $H$ to bring $y_{1}$ in normal form

$$
y_{1}=\operatorname{diag}(\underbrace{1, \ldots, 1}_{p-\text { times }}, 0, \ldots, 0) .
$$

Thus $\left(i y_{1} v, v\right)=\left(w, i y_{2} w\right)$ for some $v, w \in \mathbb{C}^{n}$ means explicitely that

$$
\left(i v_{1}, i v_{2}, \ldots, i v_{p}, 0, \ldots, 0 ; v_{1}, \ldots, v_{n}\right)=\left(w_{1}, \ldots, w_{n} ; i y_{2}(w)\right) .
$$

We conclude that $w_{p+1}=\ldots=w_{n}=0$. If $p=0$, then we are finished. So let us assume that $p>0$. But then

$$
y_{2}=\left(\begin{array}{cc}
-I_{p} & * \\
* & *
\end{array}\right)
$$

and this contradicts the fact that $y_{2}$ is positive semi-definite.

We turn our attention to the onto-ness of $p$. For that note that the closure $\operatorname{cl}(X)$ in $\mathcal{L}$ equals the geodesic compactification. As a result $\partial X=K \cdot\left(i \partial W_{d}\right)=K \cdot(i \partial W)$. Likewise $\partial \bar{X}=K \cdot(-i \partial W)$. Observe that

$$
\partial \Xi=[X \times \partial \bar{X} \amalg \partial X \times \partial \bar{X} \amalg \partial X \times \bar{X}] \cap X_{\mathbb{C}} .
$$

We first show that $X \times \partial \bar{X} \subset \operatorname{im} p$, even more precisely $p\left(G \times{ }_{H}(W \times\right.$ $\partial W))=X \times \partial \bar{X}$. In fact,

$$
X \times \partial \bar{X}=G \cdot\left(i I_{n}, K \cdot i \partial W\right)=G \cdot\left(i I_{n}, i \partial W\right)
$$

and the claim is implied by (2.1). In the manner one verifies that $\partial X \times \bar{X} \subset \operatorname{im} p$.

In order to conclude the proof it is now enough to show that $p$ is proper. This is because proper maps are closed and we have already seen that $\operatorname{im} p$ contains the dense piece $X \times \partial \bar{X} \amalg \partial X \times \bar{X} \subset \partial \Xi$. Now to see that $p$ is proper, it is enough to show that inverse images of compact subsets in $[\partial X \times \partial \bar{X}] \cap X_{\mathbb{C}}$ are compact. For the other pieces in $\partial \Xi$ this is more or less automatic: Use that $G$ acts properly on $X$, resp. $\bar{X}$ which implies that $G$ acts properly on $X \times \partial \bar{X}$ resp. $\partial X \times \bar{X}$; likewise $G$ acts properly on $G \times_{H}(W \times \partial W)$ and $G \times_{H}(\partial W \times W)$. Thus we are about to show that preimages of compacta in $[\partial X \times \partial \bar{X}] \cap X_{\mathbb{C}}$ are again compact. But this is more or less immediate from transversality; I allow myself to skip the details.

Remark 2.4. For $n=1$ the map $p$ is in fact a homeomorphism which we showed in [8]. If $n>1$, the map $p$ fails to be injective by the same computational reason shown in the preceeding remark. However, 
we emphasize that the map is generically injective and that $\left.p\right|_{\partial C}$ is injective.

\section{References}

[1] D. Akhiezer and S. Gindikin, On Stein extensions of real symmetric spaces, Math. Ann. 286 (1990), no. 1-3, 1-12.

[2] J. Faraut and A. Koranyi, Analysis on symmetric cones, Oxford Math. Monographs, Oxford University Press, New York, 1994

[3] S. Gindikin and B. Krötz, Complex crowns of Riemannian symmetric spaces and non-compactly causal symmeyric spaces, Trans. Amer. Math. Soc. 354 (8), 3299-3327

[4] S. Gindikin, B. Krötz and G. Ólafsson, Holomorphic H-spherical distribution vectors in principal series representations, Invent. math. 158, 643-682 (2004)

[5] J. Hilgert and G. Ólafsson, Causal symmetric spaces. Geometry and harmonic analysis, Perspectives in Mathematics 18, Academic Press, 1997

[6] B. Krötz, Crown theory for the upper half-plane, MPIM preprint 2007 (50), Contemp. math., to appear

[7] _ Domains of holomorphy for irreducible unitary representations of simple Lie groups, MPIM preprint 2006 (102)

[8] B. Krötz and E. M. Opdam, Analysis on the crown domain, MPIM preprint 2006 (71); to appear in GAFA

[9] B. Krötz and R. J. Stanton, Holomorphic extensions of representations: (II) geometry and harmonic analysis, GAFA, Geom. funct. anal. 15 (2005), 190245.

MaX-Planck-Institut für Mathematik, Vivatsgasse 7, D-53111 Bonn, EMAIL:KROETZ@MPIM-BONN.MPG.DE 